\title{
Crystal Structure of the FLT3 Kinase Domain Bound to the Inhibitor Quizartinib (AC220)
}

\author{
Julie A. Zorn ${ }^{1,2}$, Qi Wang ${ }^{1,2}$, Eric Fujimura ${ }^{1 \text { a }}$, Tiago Barros ${ }^{1,2,3}$, John Kuriyan ${ }^{1,2,3,4,5 *}$ \\ 1 Department of Molecular and Cell Biology, University of California, Berkeley, California, United States of \\ America, 2 California Institute for Quantitative Biosciences, University of California, Berkeley, California, \\ United States of America, 3 Howard Hughes Medical Institute, University of California, Berkeley, California, \\ United States of America, 4 Department of Chemistry, University of California, Berkeley, California, United \\ States of America, 5 Physical Biosciences Division, Lawrence Berkeley National Laboratory, Berkeley, \\ California, United States of America \\ a Current address: Department of Chemistry and Chemical Biology, Harvard University, Cambridge, \\ Massachusetts, United States of America \\ * kuriyan@berkeley.edu
}

\section{G openaccess}

Citation: Zorn JA, Wang Q, Fujimura E, Barros T, Kuriyan J (2015) Crystal Structure of the FLT3 Kinase Domain Bound to the Inhibitor Quizartinib (AC220). PLOS ONE 10(4): e0121177. doi:10.1371/journal. pone. 0121177

Academic Editor: Titus J. Boggon, Yale University School of Medicine, UNITED STATES

Received: December 10, 2014

Accepted: January 28, 2015

Published: April 2, 2015

Copyright: @ 2015 Zorn et al. This is an open access article distributed under the terms of the Creative Commons Attribution License, which permits unrestricted use, distribution, and reproduction in any medium, provided the original author and source are credited.

Data Availability Statement: All structure coordinates are deposited in the protein data bank (http://www.rcsb.org/pdb/search/structidSearch.do? structureld=4XUF).

Funding: JAZ is supported by a postdoctoral fellowship from the National Institutes of Health National Cancer Institute (F32 CA177087-02). QW is supported by an Irvington Institute postdoctoral fellowship from the Cancer Research Institute. JK and TB are funded by Howard Hughes Medical Institute. The funders had no role in study design, data collection and analysis, decision to publish, or preparation of the manuscript.

\section{Abstract}

More than $30 \%$ of acute myeloid leukemia (AML) patients possess activating mutations in the receptor tyrosine kinase FMS-like tyrosine kinase 3 or FLT3. A small-molecule inhibitor of FLT3 (known as quizartinib or AC220) that is currently in clinical trials appears promising for the treatment of AML. Here, we report the co-crystal structure of the kinase domain of FLT3 in complex with quizartinib. FLT3 with quizartinib bound adopts an "Abl-like" inactive conformation with the activation loop stabilized in the "DFG-out" orientation and folded back onto the kinase domain. This conformation is similar to that observed for the uncomplexed intracellular domain of FLT3 as well as for related receptor tyrosine kinases, except for a localized induced fit in the activation loop. The co-crystal structure reveals the interactions between quizartinib and the active site of FLT3 that are key for achieving its high potency against both wild-type FLT3 as well as a FLT3 variant observed in many AML patients. This co-complex further provides a structural rationale for quizartinib-resistance mutations.

\section{Introduction}

FMS-like tyrosine kinase 3 (FLT3) is a receptor tyrosine kinase that is expressed in both normal and malignant lympho-hematopoietic cells and is important for immune response and stem cell proliferation [1]. It belongs to a family of receptor tyrosine kinases that includes plateletderived growth factor receptors $\alpha$ and $\beta$ (PDGFR- $\alpha$ and PDGFR- $\beta$ ), colony-stimulating factor 1 (or FMS) receptor, and the stem cell factor (SCF) receptor c-Kit [2]. The characteristic domain organization of these receptors includes an extracellular module, a transmembrane (TM) helix, and an intracellular module that consists of a juxtamembrane (JM) segment, a kinase domain with a kinase insert region, and a C-terminal tail. Previous structural studies on the intracellular domains of c-Kit, FLT3, and the related vascular endothelial growth factor receptor 
Competing Interests: The authors have declared that no competing interests exist.
(VEGFR) and Eph receptor have revealed the importance of the juxtamembrane segment for stabilizing the kinase in an inactive, autoinhibited state [3-7].

The shift from an inactive to an active conformation of the kinase domain is stimulated upon ligand binding to the extracellular module to promote receptor dimerization $[8,9]$. This brings the intracellular modules into close proximity to allow the kinase domain to catalyze the transfer of a phosphate group from adenosine triphosphate (ATP) to tyrosine residues in the juxtamembrane segment of FLT3 [3]. This releases the autoinhibitory interactions and stabilizes the active kinase, which subsequently autophosphorylates additional tyrosine residues within the intracellular module of FLT3, including Tyr 842 in the activation loop to help stabilize an active conformation $[3,6]$. Phosphorylation of tyrosine residues in the C-terminal tail and the kinase insert region serve as recruitment sites for downstream substrates to initiate signaling pathways.

The deregulated activation of FLT3 due to mutation or overexpression is linked to the progression of acute myeloid leukemia (AML) and is associated with poor prognosis [10,11]. The internal tandem duplication (ITD) mutations within the juxtamembrane segment contribute to the majority of FLT3 activating mutations in AML. While this insertion can vary in length, the ITD mutations generally result in activation of FLT3 due to release of autoinhibition from the juxtamembrane segment. Additional point mutations in FLT3, which are thought to stabilize the active conformation, have also been identified in AML patients. The most prevalent of these mutations occur at Asp 835 in the activation loop. Typically, cancer cells with activated FLT3 variants become reliant on FLT3 for growth, and therefore, are susceptible to FLT3-targeted inhibitors [10,12].

For the past 20 years, drug discovery efforts have pursued the development of kinase inhibitors to block the aberrant activation of kinases associated with the cancer progression, as observed for FLT3 in AML $[13,14]$. Over 20 small molecules are now clinically approved and more than 150 additional kinase inhibitors are in clinical trials. In a recent review, we described the interactions that these clinically approved inhibitors exploit in the kinase active site [15]. In particular, FLT3 is potently inhibited by small molecules composed of a diaryl urea core scaffold, which were found to be efficacious in mouse models of the disease [16]. Chemical optimization of these compounds led to the discovery of quizartinib or AC220, which exhibits both selectivity for and potency against FLT3 [17,18]. In fact, quizartinib is currently in clinical trials and has shown promising results as a treatment for AML. However, drug resistance mutations have emerged in response to quizartinib treatment [19].

The lack of a co-crystal structure of quizartinib bound to FLT3 impedes efforts at improving FLT3 inhibitors. We now report the determination of such a structure. We had previously used modeling and molecular docking to predict how quizartinib binds to FLT3, and had found two high-scoring orientations of the drug in the kinase active site, with one preferred by the modeling procedure over the other [19]. The co-crystal structure reported here demonstrates that the drug-binding mode that was less favored in our previous modeling is the correct one. The structure also helps clarify the mechanism of drug resistance mutations, and provides a route forward for further improvement of FLT3 inhibitors.

\section{Materials and Methods}

\section{Cloning and Expression}

A vector containing the full intracellular domain of FLT3 was a gift from the Neil Shah lab at the University of California, San Francisco (UCSF). The intracellular domain of FLT3 (residues 564-994) was cloned into the pFastbacHTa (Invitrogen) insect cell expression vector, which contains an $\mathrm{N}$-terminal $\mathrm{His}_{6}$-tag with a TEV protease cleavage site. A portion of the kinase 
insert (residues 711-761) was removed from this intracellular domain construct. The recombinant virus was generated from FLT3 (564-994, $\Delta 711-761$ ) and amplified to a high titer viral stock. Sf 21 cells were grown to $2 \times 10^{6}$ cells/ $\mathrm{mL}$ and infected with virus for $48-72$ hours at $27^{\circ} \mathrm{C}$. Cells were harvested and stored at $-80^{\circ} \mathrm{C}$ until thawed for purification.

\section{Protein Purification}

Thawed cell pellet was resuspended in lysis buffer (50 mM Tris, $\mathrm{pH} 8.0$; 5\% glycerol) with protease inhibitors. The cells were lysed using a cell press, and clarified upon centrifugation at $40,000 \mathrm{rpm}$ for 90 minutes. The supernatant was filtered and loaded onto a Ni-NTA affinity column (GE Healthcare Life Sciences). The column was washed with buffer A (20 mM Tris, pH 8; $500 \mathrm{mM} \mathrm{NaCl} ; 5 \%$ glycerol), and eluted over a gradient of buffer B (20 mM Tris, pH 8; $500 \mathrm{mM} \mathrm{NaCl} ; 5 \%$ glycerol; $250 \mathrm{mM}$ imidazole). Fractions containing FLT3 were pooled; $\beta$ mercaptoethanol (Sigma-Aldrich) was added to a final concentration of $1 \mathrm{mM}$; and TEV protease was incubated with protein overnight at $4^{\circ} \mathrm{C}$. The sample was buffer exchanged into buffer $\mathrm{A}$, and a subtractive Ni-NTA column was run to remove impurities and TEV protease. The flow-through was collected, concentrated, and further purified on a Superdex $20016 / 60 \mathrm{col}-$ umn (GE Healthcare Life Sciences) in $20 \mathrm{mM}$ Tris, pH 8; $150 \mathrm{mM} \mathrm{NaCl} ; 5 \%$ glycerol. Fractions containing FLT3 were pooled, dithiothreitol (Gold Biotechnology) was added to a final concentration of $1 \mathrm{mM}$, and the sample was concentrated. Concentrated protein was aliquoted, frozen, and stored at $-80^{\circ} \mathrm{C}$.

\section{Crystallization and Structure Determination}

The FLT3 protein $(10 \mathrm{mg} / \mathrm{ml}$, in buffer containing $20 \mathrm{mM}$ Tris- $\mathrm{HCl} \mathrm{pH}$ 8.0, $150 \mathrm{mM} \mathrm{NaCl}, 1$ mM DTT and 5\% glycerol) was mixed with $0.25 \mathrm{mM}$ quizartinib (LC Laboratories) and incubated for 1 hour at room temperature. Co-crystals of FLT3-quizartinib were grown using sitting-drop vapor diffusion. $0.2 \mu \mathrm{l}$ protein stock solution was mixed with $0.2 \mu \mathrm{l}$ reservoir solution (0.1 M Tris- $\mathrm{HCl} \mathrm{pH} \mathrm{7.5,} \mathrm{32 \%} \mathrm{Polyethylene} \mathrm{glycol} \mathrm{monomethyl} \mathrm{ether} \mathrm{3360)} \mathrm{using} \mathrm{the} \mathrm{Crystal}$ Phoenix Liquid Handing system, and the drop was equilibrated at $20^{\circ} \mathrm{C}$. The crystals were cryoprotected with the addition of $20 \%$ glycerol $(\mathrm{v} / \mathrm{v})$ and X-ray diffraction data were measured at the Lawrence Berkeley National Laboratory Advanced Light Source, Beamline 8.2.2. Data were integrated and scaled using HKL2000 [20]. The dataset was anisotropic, diffracting to 2.6 $\AA$ in the a and c direction and $3.4 \AA$ in the b direction. We performed anisotropic scaling in Phenix to partially correct for the diffraction anisotropy. A molecular replacement solution was found with Phenix using two C lobes of the autoinhibited FLT3 (1RJB) as an initial search model followed by two N lobes of the autoinhibited FLT3 (1RJB) lacking the juxtamembrane segments [21]. Structure building and refinement was performed with Coot and Phenix $[21,22]$. The final model has been refined to an R-value of $29.7 \%$ (free R-value of $32.0 \%$ ) at 3.2 A resolution (see Table 1). The coordinates are deposited in the protein data bank (PDB 4XUF).

\section{Molecular Dynamics Simulations}

The autoinhibited crystal structure of FLT3 (PDB 1RJB) with and without the juxtamembrane segment present and the co-crystal structure of FLT3 with and without quizartinib present were used as the starting structures in the molecular dynamics simulations. The molecular dynamics calculations were generated using the Gromacs 4.6.2 package [23] with the ff99SB-ILDN force field [24]. The net charge on the proteins was neutralized by adding $\mathrm{Na}$ + and $\mathrm{Cl}^{-}$ions. A box of water molecules was placed on top of the crystallographic coordinates using the TIP3P water model [25]. The systems were relaxed using an initial energy 
Table 1. Structure determination and refinement of FLT3 bound to quizartinib.

\begin{tabular}{|c|c|}
\hline \multicolumn{2}{|l|}{ Data collection } \\
\hline & Native \\
\hline Wavelength $(\AA)$ & 1.0000 \\
\hline Space group & $\mathrm{P} 22_{1} 2_{1} 2_{1}$ \\
\hline \multicolumn{2}{|l|}{ Cell dimensions } \\
\hline$a, b, c(\AA)$ & $48.73,75.49,153.97$ \\
\hline Resolution $(\AA)$ & $50.0-3.2^{*}$ \\
\hline$R_{\text {sym }}(\%)$ & $20.9(59.7)$ \\
\hline$I / \sigma(I)$ & $4.7(2.0)$ \\
\hline$C C_{1 / 2}$ & $97.5(69.8)$ \\
\hline Completeness (\%) & $94.3(77.1)$ \\
\hline Redundancy & $3.5(2.9)$ \\
\hline Wilson B factor & 75.04 \\
\hline \multicolumn{2}{|l|}{ Refinement } \\
\hline Resolution & $46.46-3.2$ \\
\hline Reflections used & 9310 \\
\hline$R_{\text {free }}$ reflections & 932 \\
\hline$R_{\text {work }} / R_{\text {free }}$ & $0.297 / 0.320$ \\
\hline \multicolumn{2}{|l|}{ No. Atoms } \\
\hline Protein & 4333 \\
\hline Ligands & 80 \\
\hline Water & 2 \\
\hline \multicolumn{2}{|l|}{ Average Bfactors } \\
\hline Protein & 68.2 \\
\hline \multicolumn{2}{|c|}{ Root mean square deviation from ideality } \\
\hline Bonds $(\AA)$ & 0.004 \\
\hline Angles $\left({ }^{\circ}\right)$ & 0.874 \\
\hline \multicolumn{2}{|l|}{ Ramachandran statistics } \\
\hline Favored (\%) & 96.8 \\
\hline Disallowed (\%) & 0.0 \\
\hline MolProbity clash score & 12.82 \\
\hline
\end{tabular}

*Anisotropic diffraction to $2.6 \AA$ in the a and c directions and $3.4 \AA$ in the $b$ direction.

doi:10.1371/journal.pone.0121177.t001

minimization. Then, each protein system was heated to $300 \mathrm{~K}$ and was subjected to a $100 \mathrm{ps}$ equilibration at constant number, volume and temperature (NVT). Next, a short 100 ps equilibration was performed at constant number, pressure and temperature (NPT). The final simulations were performed without positional restraints under constant NPT conditions and with vrescale thermostats in Gromacs 4.6.2. Periodic boundary conditions were imposed on the protein systems. The particle-mesh Ewald summation technique was used to calculate long-range electrostatic interactions [26]. The cut-off for a van der Waals interaction was set at $1 \mathrm{~nm}$. A time step of $2 \mathrm{fs}$ was employed and the structures were stored every $2 \mathrm{ps}$.

\section{Results and Discussion}

\section{Quizartinib Binds to an Inactive Conformation of FLT3}

As seen in this FLT3 structure, the general kinase fold consists of a smaller $\mathrm{N}$-terminal lobe ( $\mathrm{N}$ lobe) and an $\alpha$-helical C-terminal lobe (C lobe) linked by a hinge segment (Fig $1 \mathrm{~A}$ and $1 \mathrm{~B}$ ). 
A
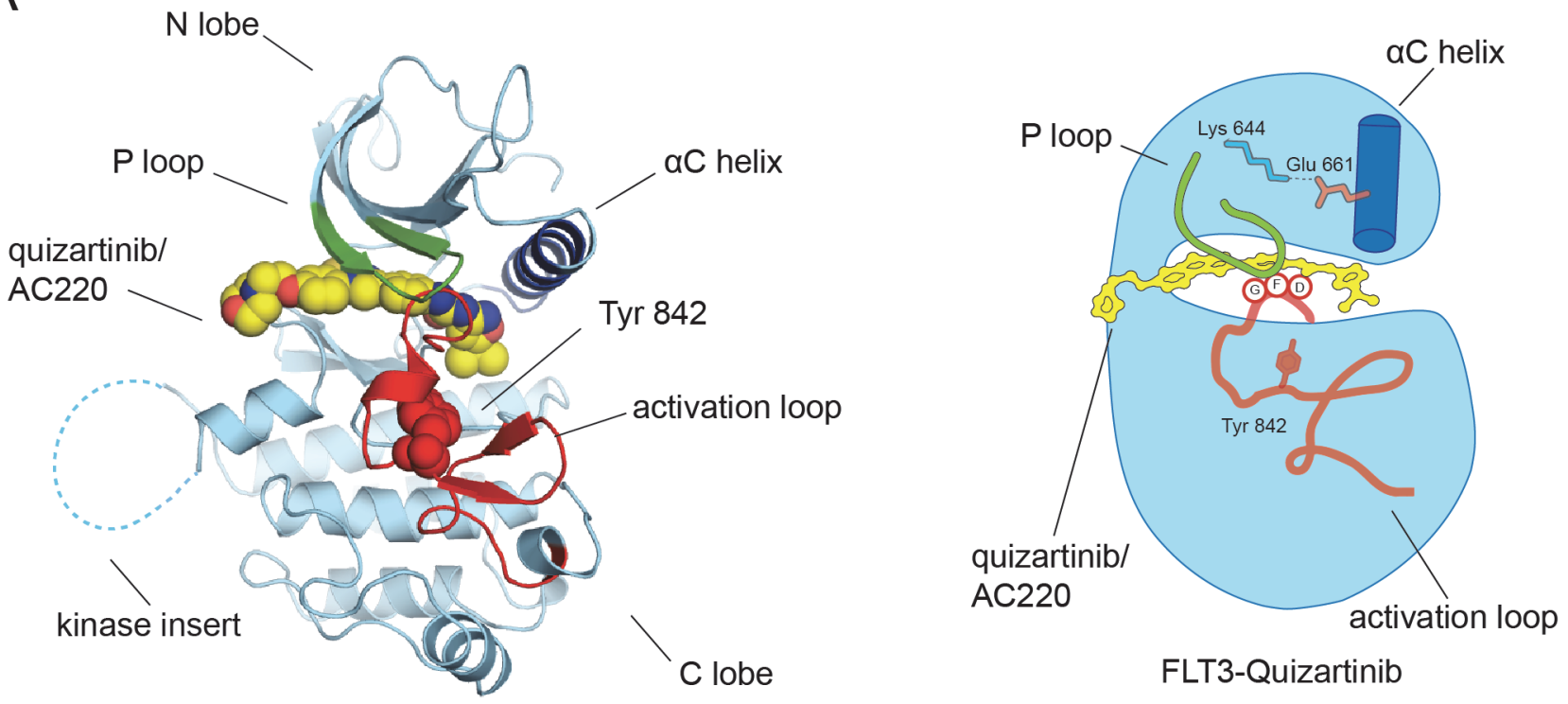

B

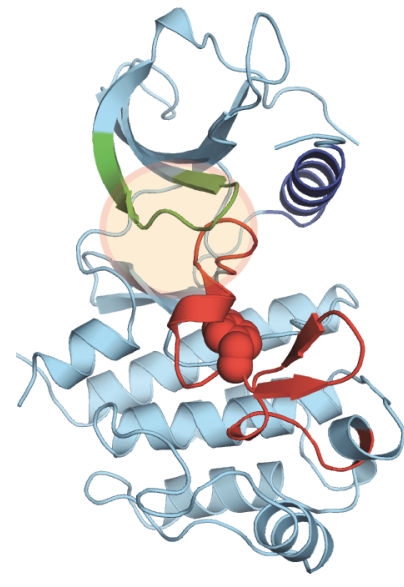

inhibited conformation quizartinib-bound structure

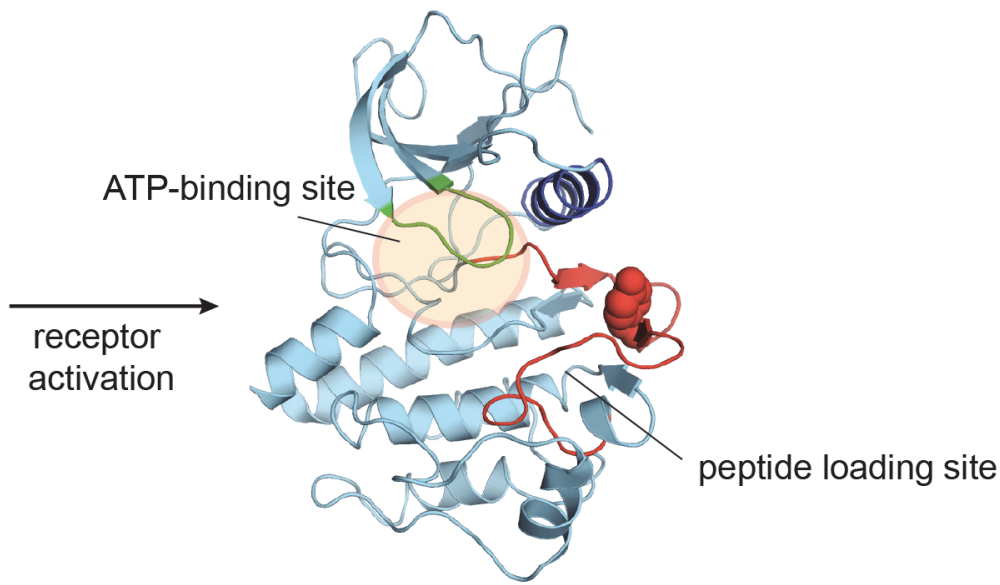

active conformation modeled from c-Kit (PDB: 1PKG)

Fig 1. FLT3 with quizartinib bound adopts an inactive conformation. (A) General kinase features are illustrated on the co-crystal structure of FLT3 bound to quizartinib. The kinase is in light blue with the $\mathrm{P}$ loop is shown in green, the $\mathrm{aC}$ helix is shown in dark blue, and the activation loop is shown in red. (B) Structural changes in kinase domain of FLT3 that occur upon receptor activation are illustrated. The active conformation of FLT3 is modeled from the active c-Kit structure (PDB 1PKG).

doi:10.1371/journal.pone.0121177.g001

The key conserved structural elements for kinase catalytic activity that lie in between the $\mathrm{N}$ lobe and the $\mathrm{C}$ lobe include the hinge region, the phosphate-binding loop (P loop), the activation loop, the catalytic loop, and the $\alpha \mathrm{C}$ helix [27].

Small molecule inhibitors have trapped many distinct conformational states of kinases. While kinases adopt a common active conformation, there are distinct inactive conformations [28]. In fact, our lab provided a structural basis for the selectivity of the first clinically approved small molecule kinase inhibitor, imatinib, bound to the tyrosine kinase, Abl [29]. Imatinib recognizes a distinct inactive kinase conformation of Abl that is also observed for many receptor tyrosine kinases, including c-Kit and VEGFR. To date, structures of kinases in complex with 
small molecules have revealed that kinases can adopt active conformations as well as diverse inactive ones [15]. The conformation of the activation loop, including that of a conserved AspPhe-Gly element, termed the DFG motif, at the base of the activation loop, and the $\alpha \mathrm{C}$ helix in kinases is typically what distinguishes these representative states.

The orientation of the DFG motif is used to classify kinase inhibitors into two representative groups. The aspartic acid residue in the DFG motif, which lies at the $\mathrm{N}$ terminus of the activation loop, must be pointed into the active site to coordinate ATP in the active conformation, which is known as the "DFG-in" conformation. Type I inhibitors are classified by this "DFGin" conformation. Type II inhibitors are defined by the "DFG-out" conformation in which the aspartate is not properly aligned for catalysis [30]. Additional features of a kinase also define the active conformation (Fig 1C). The activation loop must be extended away from the kinase domain to allow ATP and substrate to bind. The $\alpha \mathrm{C}$ helix must rotate towards the active site to permit a conserved glutamic acid residue in the $\alpha \mathrm{C}$ helix to form a salt bridge to a conserved lysine residue in the active site for ATP coordination.

In our co-crystal structure of quizartinib bound to FLT3, the kinase domain adopts an inactive conformation similar to the autoinhibited structure of FLT3 (PDB 1RJB, Fig 2A) [3]. The two structures can be overlaid very closely, with structural difference restricted to a 4-residue segment of the activation loop (residues 829-832). Excluding the activation loop (residues 829 to 858 ), the rms deviation in the C $\alpha$ positions between the two structures is $0.6 \AA$. While Glu 661 on the $\alpha \mathrm{C}$ helix forms a salt bridge with Lys 644 in the active site of FLT3, the activation loop and the DFG motif are not properly oriented to allow substrate phosphorylation to proceed. The activation loop is ordered and folded against the kinase domain to restrict access of substrate. Also, the DFG motif adopts a "DFG-out" orientation, where Phe 830 is pointed into the active site. This general kinase conformation resembles that of the inactive forms of related receptor tyrosine kinases, such as c-Kit and VEGFR, and falls into a general class termed the "Abl-like" inactive conformation [4,7,29].

The structural differences between the activation loops in autoinhibited FLT3 and the quizartinib:FLT3 co-complex appears to be induced by drug binding (Fig 2B). Even upon deleting the juxtamembrane segment, the conformation of the activation loop of the autoinhibited FLT3 remains stable in molecular dynamics simulations over $100 \mathrm{~ns}$ (Fig 2C). While the activation loop remains folded against the kinase domain in this simulation, the $\alpha \mathrm{C}$ helix does swing slightly in towards the active site. Quizartinib stabilizes a similar inactive conformation of FLT3 in molecular dynamics simulations (Fig 2D). In this case, the activation loop, the $\alpha \mathrm{C}$ helix, and the DFG motif remain stable throughout the course of the simulation. However, if quizartinib is removed from the co-crystal structure, the activation loop collapses into the active site during simulations, and Phe 830 in the DFG motif forms hydrophobic interactions with the gatekeeper residue Phe 691 (Fig 2E). The gatekeeper residue is so termed because it is a key feature of small molecule specificity determination in the kinase active site [31]. The simulations indicate that FLT3 adopts a meta-stable state in the co-crystal structure that is stabilized by quizartinib. Both the conformation of FLT3 in the co-crystal structure and the collapsed conformation adopted in the simulation after quizartinib is deleted are distinct from the conformation of the autoinhibited FLT3 (PDB 1RJB).

This FLT3 conformation with the DFG motif in the "DFG-out" orientation would characterize quizartinib as a type II inhibitor. This inhibitor class is believed to have greater selectivity relative to type I inhibitors since kinases have similar active conformations but distinct inactive conformations [32]. Consistent with our structural observations, quizartinib exhibits a high degree of potency and selectivity for FLT3 $[17,18]$. 
A

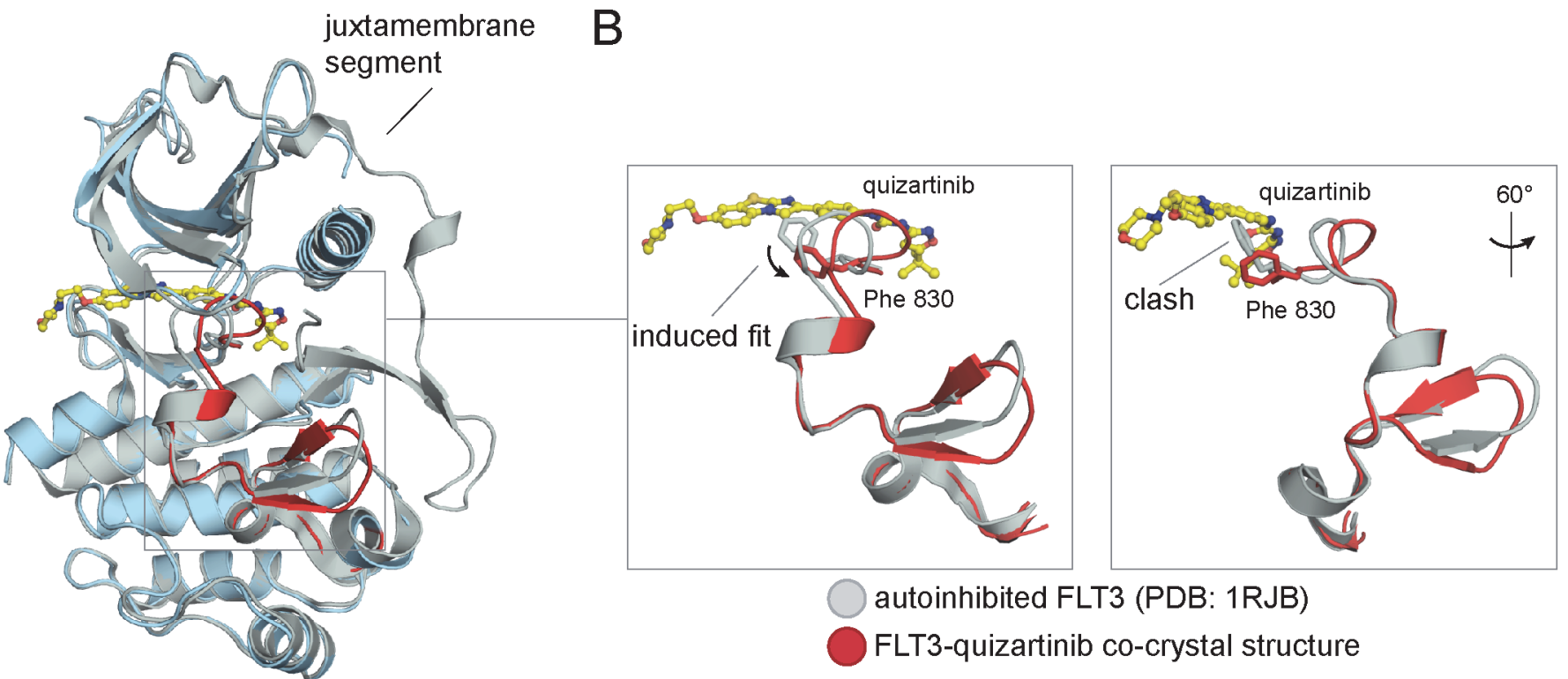

autoinhibited FLT3 (PDB: 1RJB)

(OFLT3-quizartinib co-crystal structure

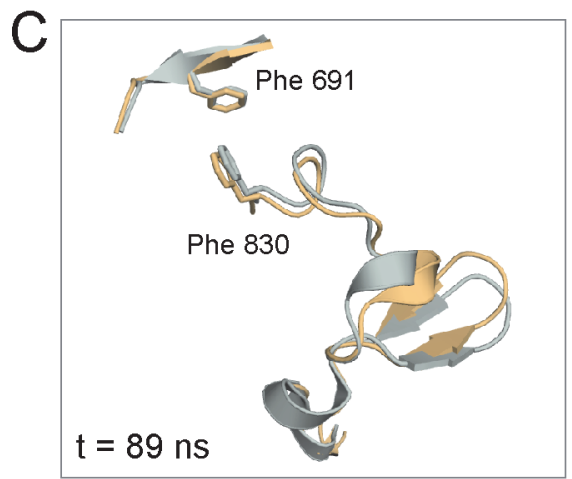

autoinhibited

FLT3 (PDB: 1RJB)

structure from simulation without JM segment

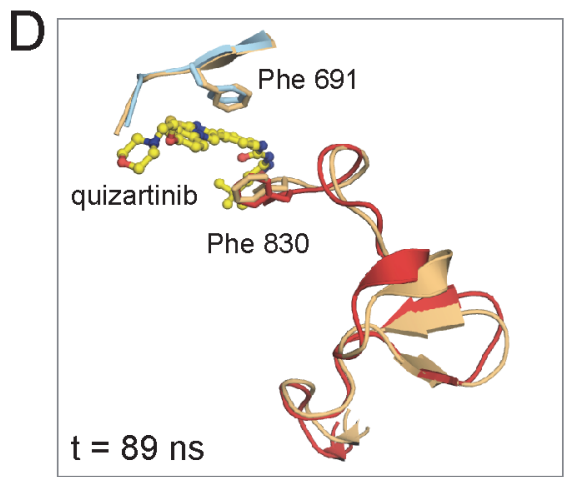

FLT3-quizartinib

co-crystal structure

structure from simulation with quizartinib

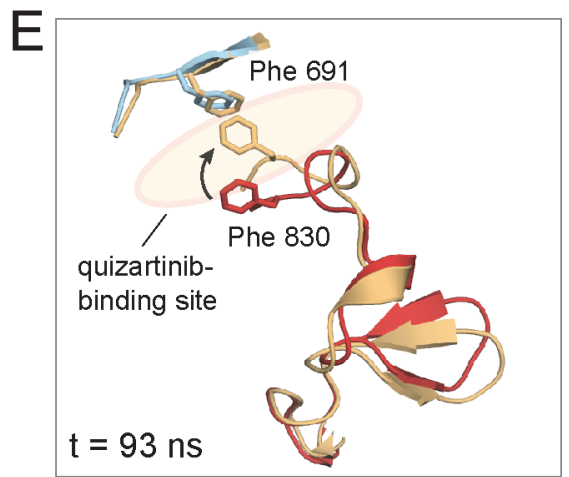

FLT3-quizartinib co-crystal structure

structure from simulation without quizartinib

Fig 2. An induced fit of the activation loop of FLT3 with quizartinib bound. (A) An overlay of the kinase domain of FLT3 from the FLT3:quizartinib cocrystal structure (using the color scheme denoted in Fig 1) with the autoinhibited FLT3 (shown in grey, PDB 1RJB). (B) A detailed view of the activation loops on both the FLT3:quizartinib (red) and the autoinhibited FLT3 (grey) structures. The left panel highlights the induced fit of Phe 830 in the DFG motif due to drug binding. Quizartinib (AC220) is included in this zoomed-in view. A $60^{\circ}$ rotation of the superposition of the two activation loops is shown in the right panel. (C) A molecular dynamics simulation on the autoinhibited FLT3 with the juxtamembrane segment deleted is illustrated with an overlay of the initial crystal structure in grey and the instantaneous structure at $t=89 \mathrm{~ns}$ in light orange. (D) The stability of the activation loop in a molecular dynamics simulation on the FLT3:quizartinib co-complex is illustrated with an overlay of the initial crystal structure in red/ blue and the instantaneous structure at $\mathrm{t}=89 \mathrm{~ns}$ in light orange. Only the chemical structure of quizartinib (yellow) from the initial structure is shown for clarity. (E) The collapse of the activation loop upon removal of quizartinib in a molecular dynamics simulation (light orange) is illustrated as a comparison to the activation loop in the co-crystal structure (red/ blue).

doi:10.1371/journal.pone.0121177.g002

\section{Interactions between quizartinib and FLT3}

Our previous report utilizing molecular docking suggest that the top-ranked binding pose of quizartinib in the active site of FLT3 is rotated $180^{\circ}$ relative to the structure determined here (Fig 3A) [19]. However, the second-ranked pose in the docking calculation is consistent with 


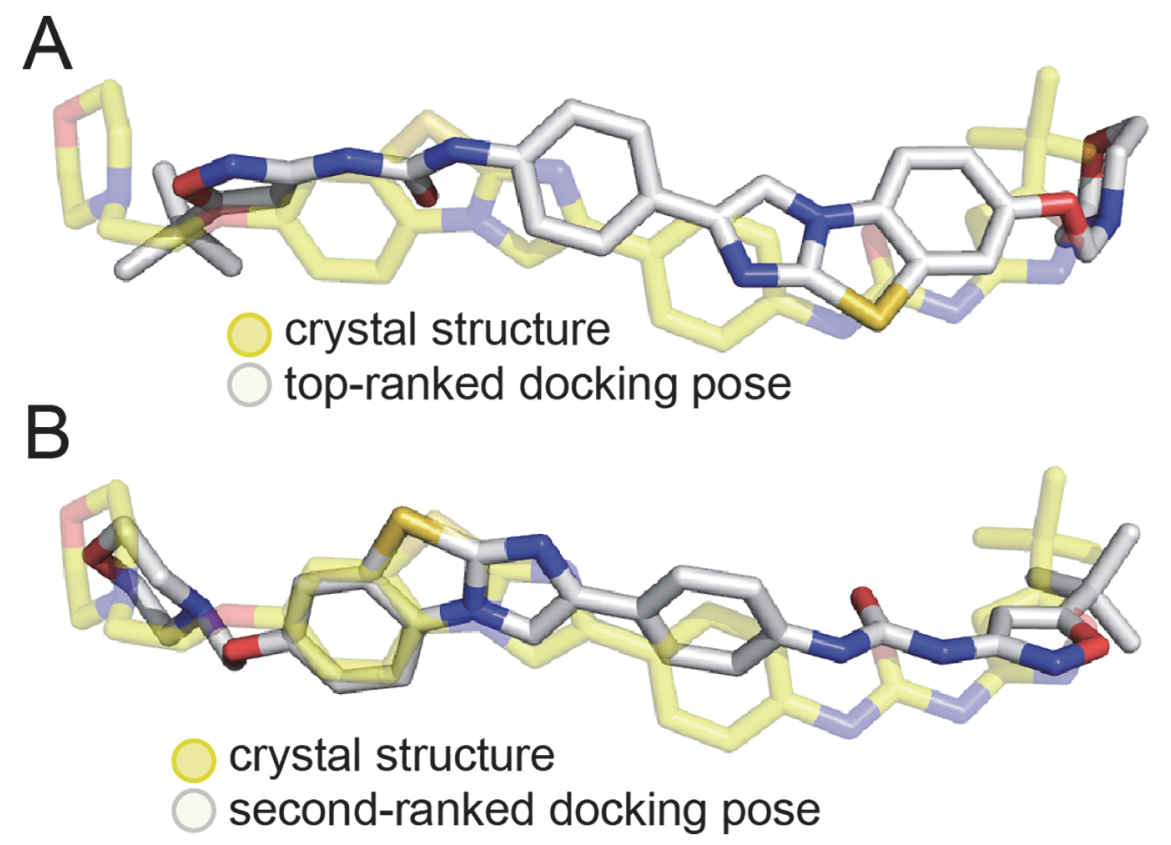

Fig 3. Quizartinib in the FLT3 co-complex matches the second-ranked docking pose. (A) An overlay of quizartinib from the co-crystal structure (yellow) with the top-ranked docking pose (white) is shown. (B) An overlay of quizartinib from the co-crystal structure (yellow) with the second-ranked docking pose (white) is shown.

doi:10.1371/journal.pone.0121177.g003

the quizartinib-FLT3 co-crystal structure (Fig 3B). An unbiased electron density map of the compound in the active site of FLT3, which was generated by performing a simulated annealing refinement on the FLT3 structure with the compound deleted, clearly reveals the orientation of quizartinib (Fig 4A and 4B).

One favorable interaction observed in the quizartinib-FLT3 co-crystal structure, in contrast to the top-ranked docking pose, is between the tert-butyl substituent on the isoxazole ring of quizartinib and a hydrophobic pocket on FLT3 (Fig 4C). The pocket includes Met 664 and Met 665 on the $\alpha \mathrm{C}$ helix, Ile 674 in the N lobe, Leu 802 in the C lobe, and Ile 827, which is immediately $\mathrm{N}$ terminal to the DFG motif (Fig 4D). Interestingly, if the co-crystal structure of VEGFR bound to sorafenib is superimposed on the co-crystal structure of FLT3 bound to quizartinib, the tert-butyl substituent on quizartinib sits in a similar pocket on FLT3 in comparison to the hydrophobic trifluoromethyl group on sorafenib in the active site of VEGFR [4]. In comparison, the top ranked docking result placed a hydrophilic morpholinoethoxy group into this pocket on FLT3. Previous structure-activity relationship studies also indicate that the morpholinoethoxy group does not affect the potency of quizartinib, but rather serves to improve its aqueous solubility [18]. This is consistent with this group pointing out of the active site into solvent, as seen in our crystal structure.

Diaryl ureas were identified as scaffolds that elicited potent inhibition of FLT3 [16]. The cocomplex of quizartinib with FLT3 reveals important interactions that this moiety makes in the kinase active site. The carbonyl group of the urea forms hydrogen bond interactions with the backbone nitrogen of Cys 828. Further, the nitrogens in the urea moiety form hydrogen bond interactions with Glu 661 in the $\alpha$ C helix (Fig 4D).

The central para-substituted phenyl ring on quizartinib makes key interactions in the kinase active site of FLT3. This ring is sandwiched between the gatekeeper residue, Phe 691, and the phenylalanine residue in the DFG motif, Phe 830 . These two residues both form $\pi-\pi$ 
A<smiles></smiles>

B

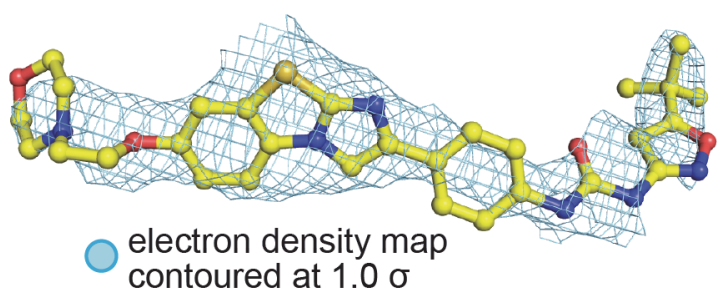

C

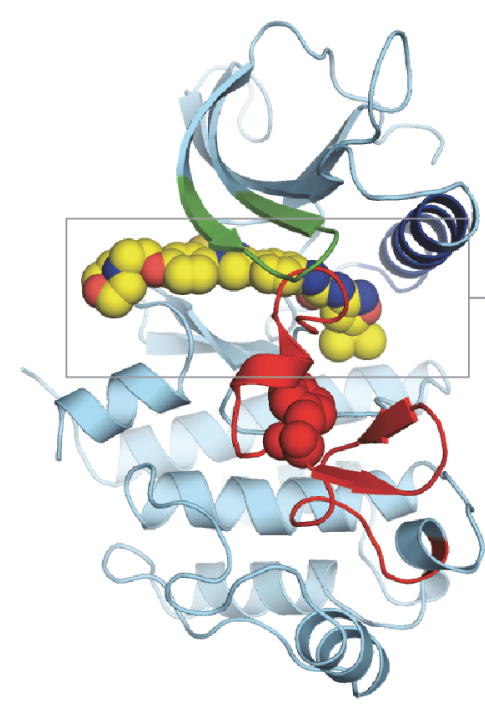

D
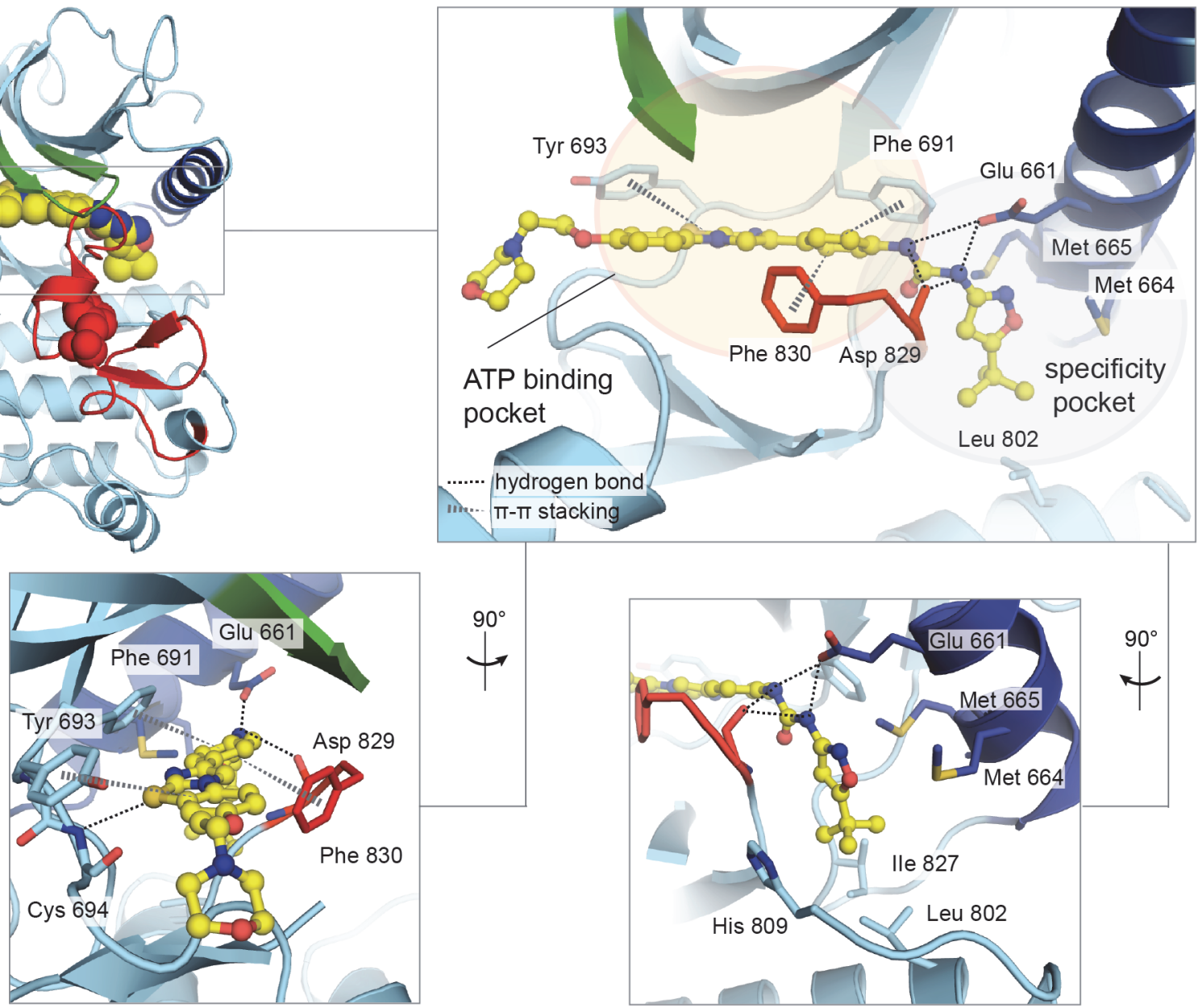

Fig 4. Interactions between FLT3 and quizartinib. (A) Chemical structure of quizartinib (AC220). (B) An unbiased electron density map (2mF $\mathrm{F}_{\mathrm{O}} \mathrm{DF} \mathrm{F}_{\mathrm{C}}$ ) of quizartinib (yellow) contoured at $1.0 \sigma$ (light blue). A simulated annealing refinement in Phenix on FLT3 with quizartinib deleted resulted in a model that was used to calculate the electron density for quizartinib. The simulated annealing was performed with an initial temperature of $5000 \mathrm{~K}$ to a final temperature of $300 \mathrm{~K}$ over 50 steps. A superposition of quizartinib with this unbiased electron density map for the compound is shown for clarity. (C) The structure of the FLT3 kinase domain bound to quizartinib (left) with a zoomed-in view of the active site (right). (D) A detailed view of the interactions between FLT3 and quizartinib.

doi:10.1371/journal.pone.0121177.g004

interactions with this ring in quizartinib, which presumably contributes to the stability of quizartinib in the active site.

The imidazobenzothiazole ring on quizartinib was previously predicted from molecular docking to form parallel $\pi-\pi$ stacking interactions with the gatekeeper residue, Phe 691 ; however, the phenyl ring of quizartinib occupies this position in the co-crystal structure, while the imidazobenzothiazole ring makes interactions with the hinge region. The sulfur atom in the 
imidazobenzothiazole ring forms a weak hydrogen bond with the backbone nitrogen of Cys 694. Additional weak $\pi-\pi$ stacking interactions between Tyr 693 in the hinge region and the imidazobenzothiazole ring are also observed in the co-crystal structure.

\section{The juxtamembrane segment of FLT3 is not compatible with quizartinib binding}

While the autoinhibited FLT3 (PDB 1RJB) and the co-crystal structure of FLT3 with quizartinib both adopt an inactive "Abl-like" structure, one distinct feature is the absence of the juxtamembrane segment in the co-crystal structure. This segment was included in the crystallization construct, but no interpretable electron density is visible for it. A superposition of the juxtamembrane segment from the autoinhibited structure onto the quizartinib-FLT3 cocomplex illustrates that quizartinib binding is not compatible with the juxtamembrane segment being folded onto the kinase domain (Fig 5A). This superposition indicates that Leu 576 on the juxtamembrane segment would clash with quizartinib. Notably, the conformation of the juxtamembrane segment in the autoinhibited FLT3 (PDB 1RJB) structure would not be compatible with the crystal packing in the FLT3:quizartinib structure, which may also contribute to the lack of electron density for this region.

Similar clashes between small molecule inhibitors and the autoinhibitory juxtamembrane segment have been reported for related receptor tyrosine kinases, including c-Kit and VEGFR. For example, the clinically-approved inhibitor imatinib is not compatible with binding of the juxtamembrane segment in c-Kit, and sorafenib is not compatible with this segment in VEGFR (Fig 5B) $[4,7]$. While these clashes have been observed for receptor tyrosine kinases, small molecule inhibitors do not always disrupt interactions between the kinase domain and the juxtamembrane segment. For example, another VEGFR inhibitor, axitinib, makes favorable interactions with the juxtamembrane segment in the kinase active site [4].

\section{Resistance mutations restrict quizartinib binding to FLT3}

Activating mutations in FLT3 are present in a significant population of AML patients, and are associated with lower response rates to traditional treatment regimens. Quizartinib is a promising therapy for these patients, but additional resistance mutations arise. One of the most common of these resistance mutations occurs at the gatekeeper residue, Phe 691, which is mutated to a leucine or isoleucine residue in patients that relapsed [33]. This mutation would disrupt a key interaction between the gatekeeper phenylalanine and a phenyl ring on quizartinib (Fig $6 \mathrm{~A})$.

Importantly, mutation of Asp 835 is not only observed as a resistance mutation to quizartinib treatment, but also as an activating mutation in patients diagnosed with AML. This residue is often mutated to hydrophobic residues, including valine, phenylalanine, and tyrosine. Asp 835 in the co-crystal structure of quizartinib bound to FLT3 does not make direct interactions with the drug. This mutation is located in the activation loop of FLT3, and may stabilize the activation loop in an active conformation, extended away from the kinase domain. Mutations in the activation loop of a kinase can alter the balance between the active and inactive state [34].

While an active conformation of FLT3 is not yet reported, an active conformation of a related family member, c-Kit, which has $65 \%$ sequence identity with the kinase domain of FLT3, has been reported (PDB 1PKG) [35]. We constructed a homology model of FLT3 using the cKit structure as a template [36]. Interestingly, Asp 835 in the model for the active conformation lies adjacent to a hydrophobic patch on FLT3 (Fig 6B). Mutation of Asp 835 to a more hydrophobic residue could promote interactions with this pocket and stabilize the activation loop in an extended conformation. In a structure of the tyrosine kinase, Lck, where the kinase domain 
A
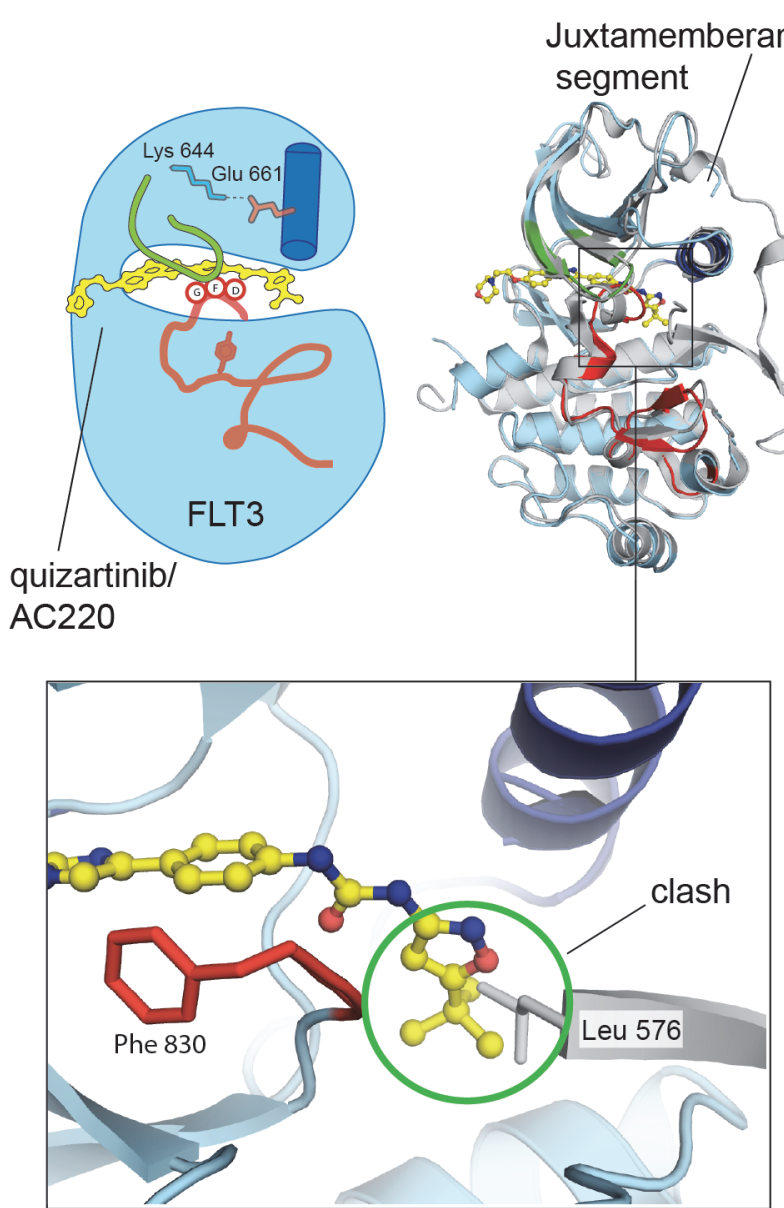

FLT3 (PDB 1RJB) and VEGFR (PDB: 4AGC)

FLT3 (this study) and VEGFR (PDB: 4ASD)
B
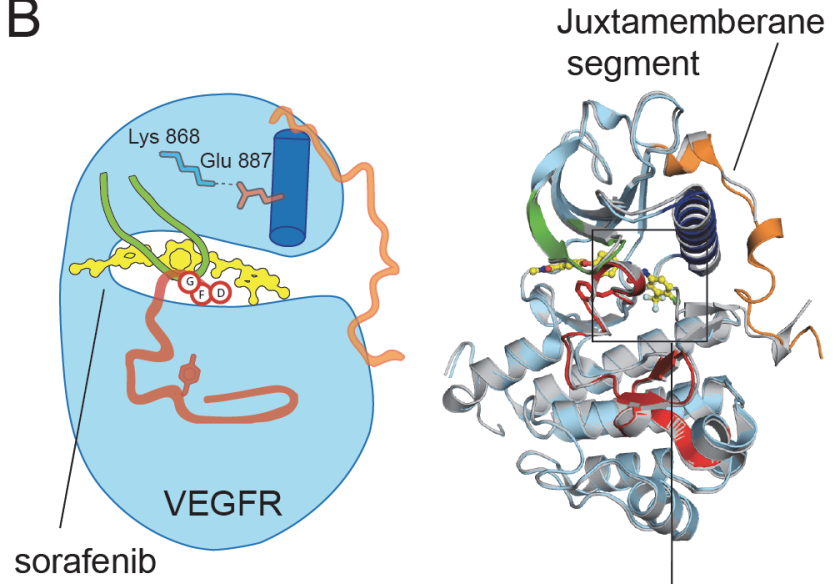

Fig 5. The juxtamembrane segment of FLT3 clashes with quizartinib. (A) An overlay of the FLT3:quizartinib crystal structure (color scheme depicted in Fig 1A) with the autoinhibited FLT3 (shown in grey, PDB 1RJB). The zoomed-in view of the juxtamembrane segment highlights clashes between the juxtamembrane segment and quizartinib. (B) An overlay of the crystal structure of the VEGFR2 kinase domain bound to sorafenib (shown in red/ blue, PDB 4ASD) and the VEGFR2 kinase domain bound to axitinib (shown in grey, PDB 4AGC). Only sorafenib is illustrated in the active site for clarity. The juxtamembrane segment from the VEGFR2:sorafenib co-crystal structure is shown in orange and is extended away from the active site. The juxtamembrane segment from the VEGFR:axitinib co-crystal structure remains bound to the kinase domain. The detailed view illustrates similar clashes between sorafenib and the VEGFR2 bound conformation of the juxtamembrane segment.

doi:10.1371/journal.pone.0121177.g005

adopts an active conformation, a leucine residue occupies this position in the activation loop and interacts with a similar hydrophobic pocket (Fig 6C) (PDB 3LCK). Mutation of this leucine residue in Lck to an aspartic acid residue results in a less active Lck variant, which is consistent with observations for FLT3 [37]. Stabilizing the active conformation of FLT3 in this fashion would disfavor quizartinib binding since the drug recognizes an inactive conformation.

\section{Conclusions}

Since receptor tyrosine kinases occupy a central role in the initiation of cellular signaling cascades, their activity often becomes deregulated in cancer $[14,38]$. Thus, many drug discovery programs have focused on the development of inhibitors targeting this family. In particular, 
A

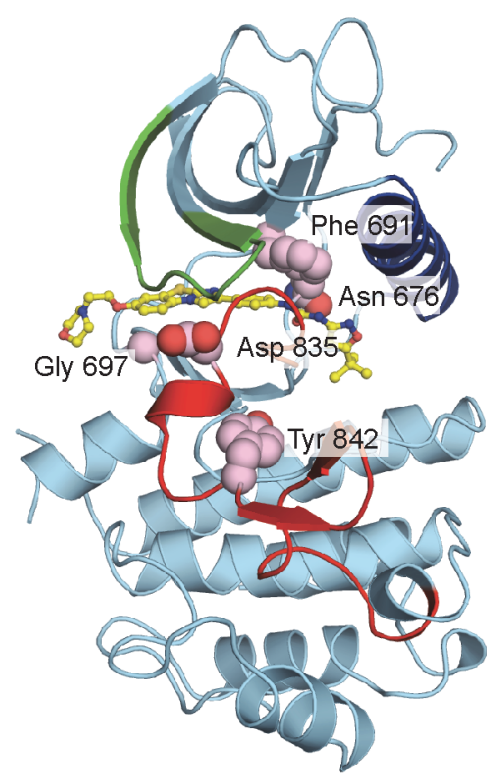

C

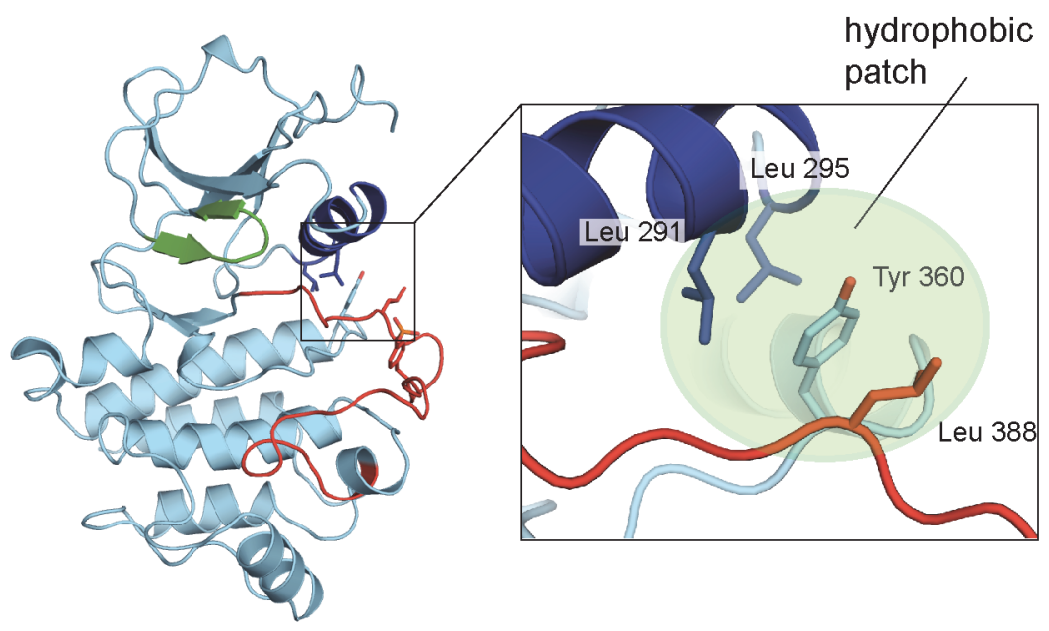

Lck (PDB: 3LCK)

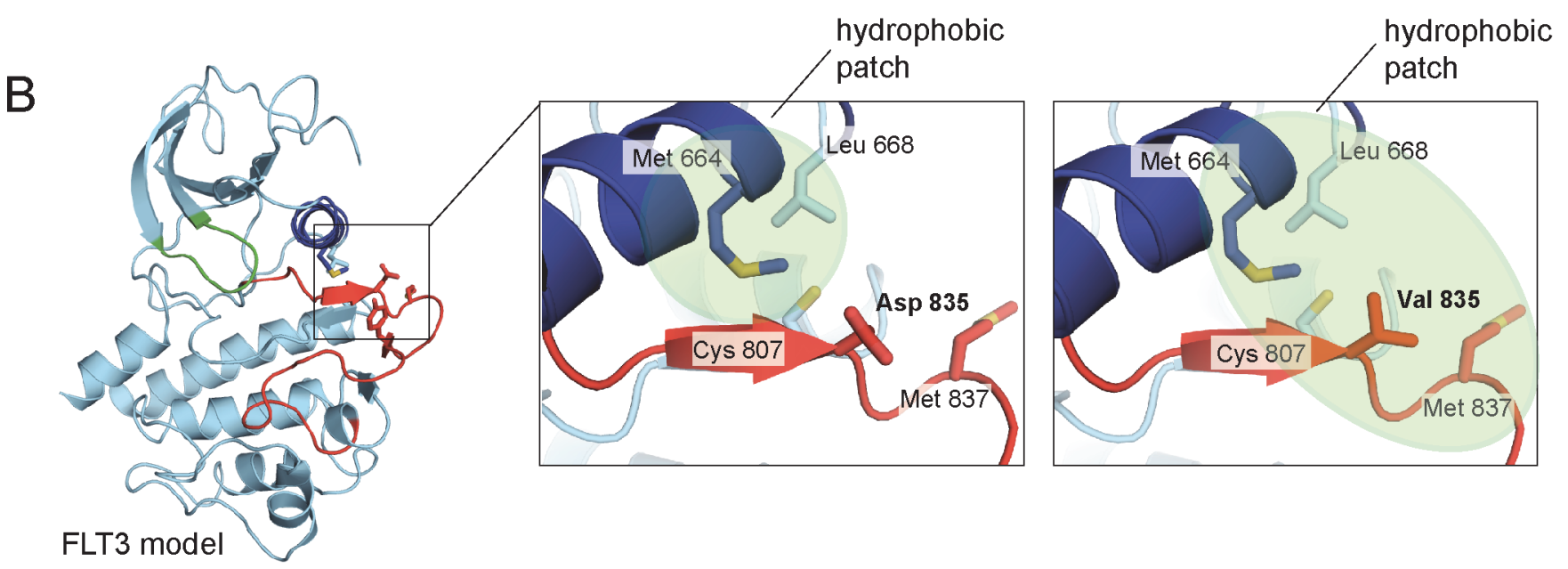

Fig 6. Mutations in the kinase domain of FLT3 in acute myeloid leukemia (AML). (A) Mutations observed in AML (pink spheres) that show resistance to quizartinib are mapped onto the kinase domain of FLT3. (B) The left panel illustrates the position of a hydrophobic pocket on a homology model of FLT3, which was generated from the active conformation of c-Kit (PDB 1PKG). The zoomed-in view in the middle panel illustrates the detailed interactions between the activation loop and a hydrophobic pocket on the model of FLT3. The right panel highlights an extension of the hydrophobic patch with the disease mutation, D835V. (C) A similar interaction between a hydrophobic pocket on the kinase domain and the activation loop is observed in the crystal structure of the active conformation of LCK (PDB 3LCK).

doi:10.1371/journal.pone.0121177.g006

FLT3 has been implicated as a driver mutation in AML [10]. Quizartinib is a second generation FLT3 inhibitor that has shown promising results in the clinic against AML.

Here, we determined the co-crystal structure of FLT3 bound to quizartinib. FLT3 adopts an "Abl-like" inactive conformation with quizartinib bound. The DFG motif adopts a "DFG-out" conformation, the activation loop is folded back onto the kinase domain to mimic peptide substrate binding, and Glu 661 on the $\alpha \mathrm{C}$ helix forms a salt bridge with Lys 644 in the active site. A previous structure of autoinhibited FLT3 shows that the juxtamembrane segment folds back onto the kinase domain and stabilizes the kinase in a similar inactive state [3]. The juxtamembrane segment, however, is not observed in our structure, and would not be compatible with quizartinib in the active site of FLT3. 
A rearrangement of the juxtamembrane segment in the autoinhibited FLT3 would be required for quizartinib to bind, similar to the rearrangement described for the related receptor tyrosine kinase, VEGFR, for sorafenib to bind [4]. In fact, quizartinib is more potent against FLT3 variants that contain the ITD activating mutations in the juxtamembrane segment, which are believed to release the inhibitory interactions that the juxtamembrane makes. Although the ITD mutation is one of the most common mutations observed in AML, restricting wild-type FLT3 activation would also be important to curb disease progression. Small molecule inhibitors that are compatible with the juxtamembrane segment conformation in the autoinhibited VEGFR were found to be more potent inhibitors relative to sorafenib [39]. Similar strategies may yield more potent and selective inhibitors against wild-type, autoinhibited FLT3.

While quizartinib is a promising treatment for AML, resistance mutations in FLT3 have been identified in response to this drug [19]. Mutation of the gatekeeper residue, F691L, and mutations in the activation loop lead to resistance. It is hoped that our structure of FLT3 with quizartinib bound will aid efforts to design new inhibitors that lead to useful therapies to treat AML patients that develop resistance to quizartinib.

\section{Acknowledgments}

We would like to thank Ann Fisher at the cell culture facility for providing insect cell cultures, and scientists at beamline 8.2.2 at the Lawrence Berkeley National Laboratory Advance Light Source for crystallography expertise. We thank Neil Shah and Catherine Smith for useful discussions.

\section{Author Contributions}

Conceived and designed the experiments: JAZ QW JK. Performed the experiments: JAZ EF. Analyzed the data: JAZ QW EF TB JK. Contributed reagents/materials/analysis tools: JAZ QW EF TB JK. Wrote the paper: JAZ QW EF TB JK.

\section{References}

1. Gilliland DG, Griffin JD. The roles of FLT3 in hematopoiesis and leukemia. Blood 2002; 100: 1532-42. PMID: 12176867

2. Lemmon MA, Schlessinger J. Cell Signaling by Receptor Tyrosine Kinases. Cell 2010; 141: 1117-34. doi: 10.1016/j.cell.2010.06.011 PMID: 20602996

3. Griffith J, Black J, Faerman C, Swenson L, Wynn M, Lu F, et al. The Structural Basis for Autoinhibition of FLT3 by the Juxtamembrane Domain. Molecular Cell 2004; 13: 169-78. PMID: 14759363

4. McTigue M, Murray BW, Chen JH, Deng Y-L, Solowiej J, Kania RS. Molecular conformations, interactions, and properties associated with drug efficiency and clinical performance among VEGFR TK inhibitors. Proceedings of the National Academy of Sciences 2012; 109: 18281-9. doi: 10.1073/pnas. 1207759109 PMID: 22988103

5. Wybenga-Groot LE, Baskin B, Ong SH, Tong J, Pawson T, Sicheri F. Structural basis for autoinhibition of the Ephb2 receptor tyrosine kinase by the unphosphorylated juxtamembrane region. Cell 2001; 106 : 745-57. PMID: 11572780

6. Hubbard SR. Juxtamembrane autoinhibition in receptor tyrosine kinases. Nat Rev Mol Cell Biol 2004; 5: 464-71. PMID: 15173825

7. Mol CD, Dougan DR, Schneider TR, Skene RJ, Kraus ML, Scheibe DN, et al. Structural basis for the autoinhibition and STI-571 inhibition of c-Kit tyrosine kinase. J. Biol. Chem. 2004; 279: 31655-63. PMID: 15123710

8. Schlessinger J. Receptor tyrosine kinases: legacy of the first two decades. Cold Spring Harbor Perspectives in Biology 2014; 6: a008912. doi: 10.1101/cshperspect.a008912 PMID: 24591517

9. Verstraete K, Vandriessche G, Januar M, Elegheert J, Shkumatov AV, Desfosses A, et al. Structural insights into the extracellular assembly of the hematopoietic Flt3 signaling complex. Blood 2011; 118: 60-8. doi: 10.1182/blood-2011-01-329532 PMID: 21389326 
10. Levis M, Small D. FLT3 tyrosine kinase inhibitors. Int J Hematol 2005; 82: 100-7. PMID: 16146839

11. Sudhindra A, Smith CC. FLT3 Inhibitors in AML: Are We There Yet? Curr Hematol Malig Rep 2014; 9 : 174-85. doi: 10.1007/s11899-014-0203-8 PMID: 24682858

12. Gunawardane RN, Nepomuceno RR, Rooks AM, Hunt JP, Ricono JM, Belli B, et al. Transient exposure to quizartinib mediates sustained inhibition of FLT3 signaling while specifically inducing apoptosis in FLT3-activated leukemia cells. Molecular Cancer Therapeutics 2013; 12: 438-47. doi: 10.1158/15357163.MCT-12-0305 PMID: 23412931

13. Cohen P, Alessi DR. Kinase drug discovery—what's next in the field? ACS Chem. Biol. 2013; 8: 96104. doi: $10.1021 / \mathrm{cb} 300610$ s PMID: 23276252

14. Blume-Jensen P, Hunter T. Oncogenic kinase signalling. Nature 2001; 411: 355-65. PMID: 11357143

15. Wang Q, Zorn JA, Kuriyan J. A structural atlas of kinases inhibited by clinically approved drugs. Meth. Enzymol. 2014; 548: 23-67. doi: 10.1016/B978-0-12-397918-6.00002-1 PMID: 25399641

16. Patel HK, Grotzfeld RM, Lai AG, Mehta SA, Milanov ZV, Chao Q, et al. Arylcarboxyamino-substituted diaryl ureas as potent and selective FLT3 inhibitors. Bioorganic \& Medicinal Chemistry Letters 2009; 19: $5182-5$.

17. Zarrinkar PP, Gunawardane RN, Cramer MD, Gardner MF, Brigham D, Belli B, et al. AC220 is a uniquely potent and selective inhibitor of FLT3 for the treatment of acute myeloid leukemia (AML). Blood 2009; 114: 2984-92. doi: 10.1182/blood-2009-05-222034 PMID: 19654408

18. Chao Q, Sprankle KG, Grotzfeld RM, Lai AG, Carter TA, Velasco AM, et al. Identification of N-(5-tertbutyl-isoxazol-3-yl)-N'-\{4-[7-(2-morpholin-4-yl-ethoxy)imidazo[2,1-b][1,3]benzothiazol-2-yl]phenyl\}urea dihydrochloride (AC220), a uniquely potent, selective, and efficacious FMS-like tyrosine kinase-3 (FLT3) inhibitor. J. Med. Chem. 2009; 52: 7808-16. doi: 10.1021/jm9007533 PMID: 19754199

19. Smith CC, Wang Q, Chin C-S, Salerno S, Damon LE, Levis MJ, et al. Validation of ITD mutations in FLT3 as a therapeutic target in human acute myeloid leukaemia. Nature 2013; 485: 260-3.

20. Otwinowski Z, Minor WC. Processing of X-ray diffraction data collected in oscillation mode. Meth. Enzymol. 1997; 276: 307-26.

21. Adams PD, Afonine PV, Bunkóczi G, Chen VB, Davis IW, Echols N, et al. PHENIX: a comprehensive Python-based system for macromolecular structure solution. Acta Crystallogr. D Biol. Crystallogr. 2010; 66: 213-21. doi: 10.1107/S0907444909052925 PMID: 20124702

22. Emsley P, Cowtan K. Coot: model-building tools for molecular graphics. Acta Crystallogr. D Biol. Crystallogr. 2004; 60: 2126-32. PMID: 15572765

23. Pronk S, Páll S, Schulz R, Larsson P, Bjelkmar P, Apostolov R, et al. GROMACS 4.5: a high-throughput and highly parallel open source molecular simulation toolkit. Bioinformatics 2013; 29: 845-54. doi: 10 1093/bioinformatics/btt055 PMID: 23407358

24. Lindorff-Larsen K, Piana S, Palmo K, Maragakis $P$, Klepeis JL, Dror RO, et al. Improved side-chain torsion potentials for the Amber ff99SB protein force field. Proteins 2010; 78: 1950-8. doi: 10.1002/prot. 22711 PMID: 20408171

25. Jorgensen WL. Quantum and statistical mechanical studies of liquids. 10. Transferable intermolecular potential functions for water, alcohols, and ethers. Application to liquid water. J. Am. Chem. Soc. 1981; 103: 335-40.

26. Darden T, York D, Pedersen L. Particle mesh Ewald: An N. $\log (\mathrm{N})$ method for Ewald sums in large systems. The Journal of chemical physics 1993; 98: 10089.

27. Schwartz PA, Murray BW. Protein kinase biochemistry and drug discovery. Bioorg. Chem. 2011; 39: 192-210. doi: 10.1016/j.bioorg.2011.07.004 PMID: 21872901

28. Huse M, Kuriyan J. The conformational plasticity of protein kinases. Cell 2002; 109: 275-82. PMID: 12015977

29. Schindler T, Bornmann W, Pellicena P, Miller WT, Clarkson B, Kuriyan J. Structural mechanism for STI-571 inhibition of abelson tyrosine kinase. Science 2000; 289: 1938-42. PMID: 10988075

30. Zhao Z, Wu H, Wang L, Liu Y, Knapp S, Liu Q, et al. Exploration of Type II Binding Mode: A Privileged Approach for Kinase Inhibitor Focused Drug Discovery? ACS Chem. Biol. 2014; 9: 1230-41. doi: 10. 1021/cb500129t PMID: 24730530

31. Schindler T, Sicheri F, Pico A, Gazit A, Levitzki A, Kuriyan J. Crystal structure of Hck in complex with a Src family-selective tyrosine kinase inhibitor. Molecular Cell 1999; 3: 639-48. PMID: 10360180

32. Liu Y, Gray NS. Rational design of inhibitors that bind to inactive kinase conformations. Nature Chemical Biology 2006; 2: 358-64. PMID: 16783341

33. Albers C, Leischner H, Verbeek M, Yu C, Illert AL, Peschel C, et al. The secondary FLT3-ITD F691L mutation induces resistance to AC220 in FLT3-ITD+ AML but retains in vitro sensitivity to PKC412 and Sunitinib. Leukemia 2013; 27: 1416-8. doi: 10.1038/leu.2013.14 PMID: 23392356 
34. Young MA, Shah NP, Chao LH, Seeliger M, Milanov ZV, Biggs WH, et al. Structure of the kinase domain of an imatinib-resistant Abl mutant in complex with the Aurora kinase inhibitor VX-680. Cancer Research 2006; 66: 1007-14. PMID: 16424036

35. Mol CD, Lim KB, Sridhar V, Zou H, Chien EYT, Sang B-C, et al. Structure of a c-kit product complex reveals the basis for kinase transactivation. J. Biol. Chem. 2003; 278: 31461-4. PMID: 12824176

36. Smith CC, Lasater EA, Lin KC, Wang Q, McCreery MQ, Stewart WK, et al. Crenolanib is a selective type I pan-FLT3 inhibitor. Proceedings of the National Academy of Sciences 2014; 111: 5319-24. doi: 10.1073/pnas.1320661111 PMID: 24623852

37. Laham LE, Mukhopadhyay N, Roberts TM. The activation loop in Lck regulates oncogenic potential by inhibiting basal kinase activity and restricting substrate specificity. Oncogene 2000; 19: 3961-70. PMID: 10962552

38. Levitzki A, Mishani E. Tyrphostins and other tyrosine kinase inhibitors. Annu. Rev. Biochem. 2006; 75: 93-109. PMID: 16756486

39. Solowiej J, Bergqvist S, McTigue MA, Marrone T, Quenzer T, Cobbs M, et al. Characterizing the effects of the juxtamembrane domain on vascular endothelial growth factor receptor-2 enzymatic activity, autophosphorylation, and inhibition by axitinib. Biochemistry 2009; 48: 7019-31. doi: 10.1021/ bi900522y PMID: 19526984 